\title{
Construção e validação da Escala Multifatorial de Atração por Eventos Mórbidos
}

\author{
Construction and Validation of \\ Attraction for Morbid Events Multifactor Scale \\ Lia Plutarco'
(D) https://orcid.org/0000-0001-6185-1552 \\ Marília Mendes Moreira de Sousa ${ }^{2}$ \\ (1) https://orcid.org/0000-0002-0114-5602 \\ Walberto Santos ${ }^{3}$ \\ https://orcid.org/0000-0001-6816-0105 \\ Sophia Sousa ${ }^{4}$ \\ https://orcid.org/0000-0002-1470-5867 \\ Matheus Gomes Lins Alves 5 \\ Dttps://orcid.org/0000-0003-0113-2980 \\ 1,2,3,4,5 Departamento de Psicologia, Universidade Federal do Ceará, Brasil
}

Resumo. Objetivo. Este estudo buscou construir e validar a Escala Multifatorial de Atração por Eventos Mórbidos (AMAEM). Método. Do primeiro estudo participaram 252 pessoas da população geral, as quais responderam à EMAEM e a perguntas sociodemográficas. A análise de componentes principais indicou uma estrutura de cinco fatores. No segundo estudo, a amostra foi de 609 pessoas da população geral brasileira, as quais a escala de personalidade e a escala de busca de sensações. Resultado. A análise fatorial confirmatória apoaiou a adequação do modelo de cinco fatores. Adicionalmente, demonstrou ainda que os fatores da EMAEM podem contribuir com a compreensão de características da personalidade e sua associação com o engajamento em comportamentos antissociais.

Palavras-chave. Personalidade, estrutura fatorial, busca de sensações, comportamento antissocial, comportamento de risco

Abstract. Objective. This study aimed to construct and validate the Attraction for Morbid Events Multifactor Scale (EMAEM) Method. Two empiric studies were conducted. 252 people from the general population participated in the first study, in which a main component analysis was conducted to attest the factor structure of EMAEM. In this study, the five-factor model was most suitable. In the second study, the participants were 609 people from the general population that answered personality and antisocial behavior scales, besides EMAEM. Results. Confirmatory Factor Analysis shows the suitability of the five-factor model. Moreover, the EMAEM scale can provide aids to a better understanding of personality traits associated with engaging in risky behavior.

Keywords. Personality, factor structure, sensation seeking, antisocial behavior, risky behavior

\footnotetext{
${ }^{1}$ Lia Plutarco. Departamento de Psicologia, Universidade Federal do Ceará, Brasil. Direção postal: Avenida da Universidade, 2853, Benfica, 60020-181, Fortaleza - CE, Brasil. E-mail: liaplutarco@hotmail.com

2Marília Mendes Moreira de Sousa. Departamento de Psicologia, Universidade Federal do Ceará, Brasil. E-mail: mariliamendesms@gmail.com ${ }^{3}$ Walberto S. Santos. Departamento de Psicologia, Universidade Federal do Ceará, Brasil. E-mail: walbertosantos@ufc.br ${ }^{4}$ Sophia Lóren de Holanda Sousa. Departamento de Psicologia. Brasil. E-mail: sophialorensh1@gmail.com

${ }^{5}$ Matheus Gomes Lins Alves. Departamento de Psicologia. Brasil. E-mail: matheus_gla@hotmail.com
}

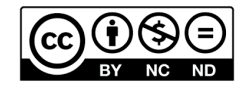

Esta obra está bajo una licencia de Creative Commons Reconocimiento-NoComercial-SinObraDerivada 4.0 Internacional. 


\section{Introdução}

A curiosidade pode ser definida como um desejo pelo conhecimento que motiva os indivíduos a aprenderem novas ideias, eliminarem brechas na informação entre aquilo que sabem e o que gostariam de saber (information-gaps) e resolverem problemas intelectuais (Litman, 2005; Hsee \& Ruan, 2016). Ainda que a curiosidade esteja relacionada a processos evolutivamente considerados vantajosos, como a aprendizagem (Gruber et al., 2014), ela também pode ser percebida como uma desvantagem, visto que a procura por informações pode ocorrer a despeito de prováveis resultados indesejados (Hsee \& Ruan, 2016).

Desse modo, é possível que as pessoas explorem estímulos sobre morte ou violência devido à possibilidade de obterem ferramentas para lidar com eventos negativos futuros (Oosterwijk, 2017). Com efeito, na pesquisa de Baumeister et al. (2001), os autores encontraram que eventos negativos têm um maior impacto psicológico (e.g. melhor elaboração cognitiva) quando comparados a eventos neutros ou positivos. Conclui-se, então, que ser mais sensível a informações negativas é evolutivamente vantajoso.

No Brasil, o consumo atual de programas de televisão parece concordar com a premissa apresentada. Em um levantamento feito pela Secretaria Especial de Comunicação do Governo Federal, encontrou-se que 63\% da população do país elegeu a TV como meio de comunicação preferido para obter informação diariamente (Brasil, 2016). Com base nessa estatística e no conteúdo exibido pela TV aberta, é possível conjecturar que o brasileiro assiste com frequência a programas cujos conteúdos expressam cenas ou relatos de violência. No estado do Ceará, por exemplo, um dos jornais de grande porte (Narlla, 2015) noticiou a consolidação do programa "Barra Pesada" como um dos mais populares do estado, marcando 17 pontos de audiência. Tal programa tem como foco principal de suas matérias eventos associados à violência urbana.

No entanto, apesar do consumo de mídia violenta ser alto no Brasil, o tema se mostra pouco estudado. Em especial, no campo da psicologia, destaca-se que algumas pessoas possuem um traço de personalidade denominado curiosidade por eventos mórbidos. Zuckerman (2007) define a curiosidade por eventos mórbidos como uma necessidade por experiências despertadoras de modo geral, que implica no desejo de presenciar ou ler sobre eventos que promovam altos níveis de sensação, como consumir conteúdos midiáticos violentos, a preferência por esportes violentos, por filmes de terror e suspense, a excitação ao presenciar um acidente de trânsito, dentre outros.

A literatura existente acerca da curiosidade por eventos mórbidos é escassa e muitas das pesquisas sobre o tema são antigas. Zuckerman e Litle (1986) foram um dos precursores no estudo desse tema e encontraram que esse traço de personalidade é associado com aspectos da personalidade desviante, como a busca de sensações. Os autores encontraram que buscadores de sensação pontuaram alto na Escala de Curiosidade Sobre Eventos Mórbidos (Curiosity About Morbid Events - CAME).

Zuckerman (2007) afirma que a busca de sensações envolve além da procura por sensações variadas e intensas, a disposição em expor-se a riscos para que uma experiência nova seja alcançada. De fato, pessoas com alta pontuação em busca de sensações são mais agressivas (Zuckerman et al., 1993), mais curiosas sobre os eventos mórbidos (Zuckerman \& Litle, 1986), mais atraídas por comportamentos perigosos (Roberti, 2004) e mais propensas a cometer um crime não violento (Horvath \& Zuckerman, 1993). Pode-se conjecturar então que a curiosidade por eventos mórbidos, por estar relacionada ao traço de busca 
de sensações, até mesmo em sua definição, também se encontre relacionada com a maior probabilidade de engajamento em comportamentos de risco ou antissociais.

Além disso, no estudo da personalidade, a curiosidade por eventos mórbidos também se encontra associada ao modelo de três dimensões primárias da personalidade (Eysenck \& Eysenck, 1985). O modelo dos cinco grandes fatores de personalidade (John et al., 1991), que estrutura a personalidade por meio dos fatores neuroticismo, extroversão, amabilidade, conscienciosidade e abertura a mudanças, também pode ser correlacionado com a atração por eventos mórbidos (Pimentel et al., 2014). Pimentel et al. (2014) apontam que amabilidade e conscienciosidade correlacionam-se negativamente com a preferência por filmes de terror. No caso de abertura a mudanças, extroversão e neuroticismo a correlação foi positiva, indicando assim uma preferência pelo gênero. Ademais, um aspecto interessante a ser observado é que o construto parece manter-se estável em pesquisas transculturais (Agnieszka, 2018; Weaver III et al., 1993; Zuckerman \& Litle, 1986).

O objetivo do presente artigo é propor, com base na Escala de Curiosidade Sobre Eventos Mórbidos (CAME), a Escala Multifatorial de Atração por Eventos Mórbidos (EMAEM). Especificamente, espera-se, além de adaptar e construir seus itens, apresentar indicadores de validade e precisão, atestando a adequabilidade da medida para utilização em contexto brasileiro.

\section{Estudo 1}

\section{Elaboração e análise de componentes principais da EMAEM}

\section{Método}

\section{Amostra}

Contou-se com a participação de 252 pessoas da população geral, divididas quase equitativamente entre sexo (56.9\% do sexo masculino), com idades variando entre 18 e 80 anos $(M=31.67 ; D P=14.39)$, cuja maioria afirmou pertencer à religião católica (38.9\%), ser solteira (59.9\%), heterossexual (82.4\%) e ter ensino superior incompleto (29.4\%).

\section{Instrumentos}

Escala Multifatorial de Atração por Eventos Mórbidos (EMAEM). Foi criada com base na Curiosity About Morbid Events Scale, desenvolvida por Zuckerman e Litle (1986), a qual reflete o interesse em assistir violência ou eventos mórbidos em esportes, filmes, TV, ou em outros eventos no mundo real. A escala original é composta por 20 itens (e.g. "Eu gostaria de ver uma execução"; ou "Gosto de sentir-me levemente assustado pelos filmes de terror"), dos quais sete são invertidos e indicam aversão ou evitação dessas experiências. Na escala adaptada para esse estudo, utilizou-se na íntegra apenas o item "Filmes de terror me deixam empolgado". Os demais itens foram transformados ou criados de forma que se enquadrassem melhor ao contexto estudado, assim como aos programas televisivos e às tecnologias atuais. O item original "Eu gostaria de ver a realização de uma autópsia", foi modificado para a versão "Seria interessante assistir a uma autópsia (médicos examinando um cadáver aberto)", por exemplo. A versão final da escala aplicada aos participantes foi formada por 30 itens, os quais descrevem diversas situações de eventos mórbidos reais ou fictícios. Ressalta-se que essa versão passou por uma análise de juízes, de forma a obter itens adequados avaliados por peritos.

Questionário de caracterização sociodemográfica. Para fins de caracterizar a amostra, foi requisitado aos participantes que respondessem algumas questões acerca de dados 
pessoais, como idade, religião, orientação sexual, escolaridade, dentre outros.

Procedimentos de coleta de dados

Os dados foram coletados por meio de um questionário online e por questionários impressos, aplicados em locais públicos. Os sujeitos concordaram com sua participação por meio do Termo de Consentimento Livre e Esclarecido (TCLE). Foi garantido por meio do TCLE, de acordo com a Resolução nº 016/2000 artigo $4^{\circ}$ do Conselho Federal de Psicologia (CFP, 2000), a participação voluntária do sujeito, o esclarecimento sobre os objetivos da pesquisa e o uso que será feito das informações recolhidas.

Procedimentos de análise de dados

Para avaliar a estrutura fatorial da EMAEM foi realizada uma análise de componentes principais utilizando o software SPSS 21.

\section{Resultados}

Inicialmente, foi averiguada a adequabilidade da matriz de dados para realização de uma análise de componentes principais. Para tanto, observaram-se o Kaiser-Meyer-Olkin (KMO $=0.83$ ) e o Teste de Esfericidade de Bartlett $(435)=2865,30, p<.001$ ), cujos resultados indicaram a pertinência da matriz para efetuar as análises. Em seguida, foi analisado o número possível de fatores a serem extraídos da matriz. Nessa etapa, foram observados o critério de Kaiser - ou eigenvalue (autovalor) superior a 1, cujo resultado indicou ser possível a extração de até oito fatores; o scree plot (Figura 1), que demonstrou ser adequado extrair até seis fatores da matriz de dados; e a análise paralela, que demonstrou ser mais adequada a extração de até cinco fatores da matriz de dados original.

Com base nos resultados, optou-se por realizar uma análise de componentes principais, com rotação oblimin e fixando a extração de cinco fatores, visto que a análise paralela pode ser considerada o critério mais robusto para definir o número de fatores a serem extraídos da matriz (Hayton et al., 2004). Os resultados podem ser observados na Tabela 1.

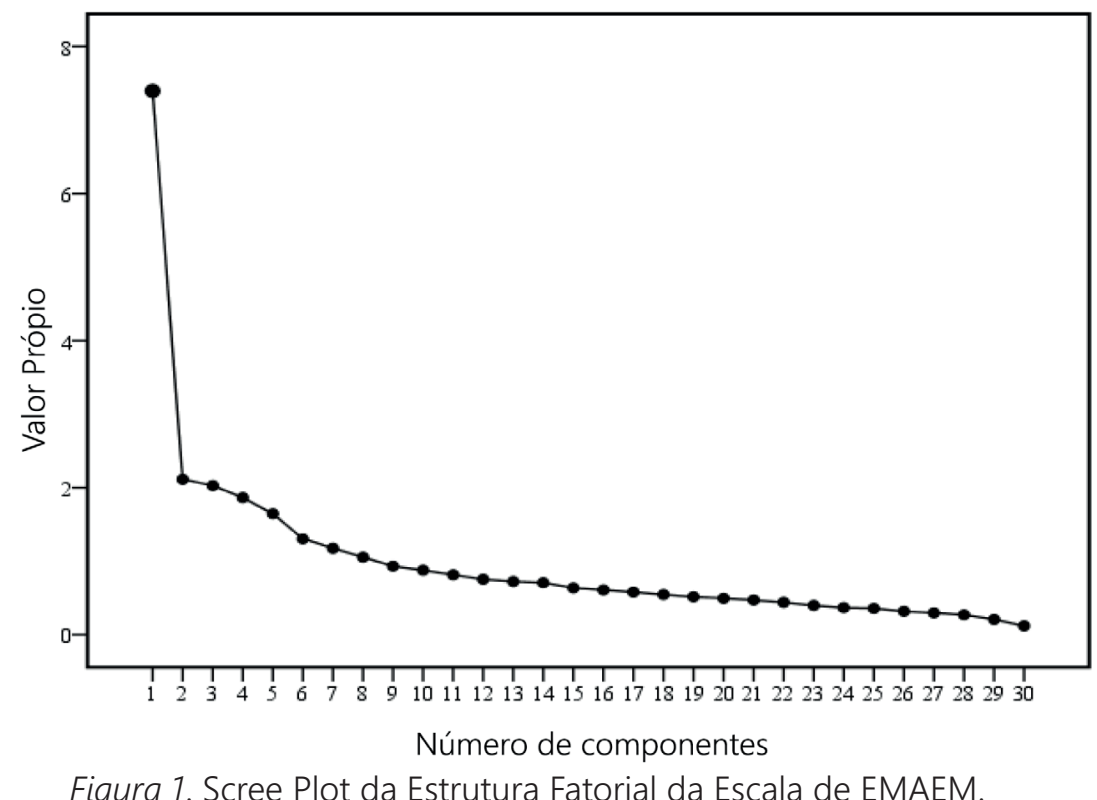

Actualidades en Psicología, 35(131), 2021, 103-118 
Tabela 1

Cargas Fatoriais, comunalidade e variância explicada pela Escala Multifatorial de Atração por Eventos Mórbidos $(n=262)$

\begin{tabular}{|c|c|c|c|c|c|c|}
\hline Conteúdo dos itens & $\mathrm{F} 1$ & $\mathrm{~F} 2$ & $\mathrm{~F} 3$ & $\mathrm{~F} 4$ & F5 & $h^{2}$ \\
\hline \multicolumn{7}{|l|}{ Fator 1: "Eventos mórbidos reais" } \\
\hline 02. Eu assistiria, sem problemas, a duas pessoas... & .37 & -.33 & .14 & -.02 & .07 & .14 \\
\hline 23. Não sei o porquê, mas fico muito empolgado... & .46 & -.06 & .30 & -.07 & .07 & .21 \\
\hline 08. Cenas reais de pessoas pulando das janelas... & .55 & .04 & .06 & -.22 & .01 & .30 \\
\hline 14. Sinto curiosidade em ver cenas de terroristas... & .35 & .02 & .21 & -.01 & .06 & .12 \\
\hline 15. Notícias de ataques de animais a seres humanos... & .40 & -.14 & .27 & -.22 & -.18 & .16 \\
\hline 16. Se tivesse a oportunidade de ver alguém... & .68 & .01 & -.05 & .08 & .03 & .46 \\
\hline 17. Deve ser excitante presenciar uma execução de... & .71 & -.08 & -.09 & .16 & .11 & .50 \\
\hline 27. Fico empolgado(a) quando assisto a espetáculos... & .35 & -.12 & .27 & -.01 & .12 & .12 \\
\hline 24. Caso visse uma pessoa tentando atirar em outra... & .75 & .04 & -.07 & .02 & .04 & .56 \\
\hline 18. Saber que uma pessoa acabou de se jogar de um... & .57 & -.01 & -.11 & -.45 & -.16 & .32 \\
\hline \multicolumn{7}{|l|}{ Fator 2: "Aversão a Eventos Mórbidos" } \\
\hline 01. Se percebo que um vídeo compartilhado nas... & -.19 & .41 & .04 & -.01 & -.34 & .17 \\
\hline 13. Fico angustiado(a) só de pensar na cena de um... & -.06 & .62 & .32 & -.01 & -.01 & .38 \\
\hline 20. Sinto-me incomodado com programas que... & -.03 & .53 & -.01 & -.13 & -.40 & .28 \\
\hline 28. Em cenas sangrentas de filmes violentos costumo... & -.04 & .62 & -.22 & .07 & .20 & .38 \\
\hline 30. Fico agoniado(a) quando estou assistindo a uma... & -.01 & .61 & -.14 & .01 & .04 & .37 \\
\hline 10. Em filmes, cenas de cadáveres amontoados me... & .01 & .67 & -.20 & .00 & .04 & .45 \\
\hline 12. Eu ficaria incomodado em me aproximar do... & .14 & .53 & .26 & .38 & -.09 & .28 \\
\hline \multicolumn{7}{|l|}{ Fator 3: "Eventos Mórbidos Fictícios" } \\
\hline 29. Filmes de terror me deixam empolgado(a). & -.07 & -.12 & .79 & -.09 & .02 & .62 \\
\hline 22. Histórias de crimes e de assassinatos despertam... & .19 & .09 & .52 & -.24 & .04 & .27 \\
\hline 03. Gosto de filmes que misturam terror e violência. & .04 & -.19 & .77 & -.01 & .04 & .59 \\
\hline 05. Poucas coisas são mais empolgantes do que um... & -.06 & -.07 & .42 & .05 & .52 & .18 \\
\hline \multicolumn{7}{|l|}{ Fator 4: "Eventos mórbidos Médicos" } \\
\hline 19. Acidentes com fraturas expostas e pedaços... & .38 & -.01 & .00 & -.54 & -.14 & .29 \\
\hline 25. Seria interessante assistir a uma autópsia... & -.10 & .05 & .16 & -.88 & .05 & .77 \\
\hline 26. Gostaria de assistir a uma cirurgia em que o... & -.11 & .01 & .09 & -.89 & .04 & .79 \\
\hline 07. Deve ser interessante presenciar o SAMU... & .20 & -.18 & -.14 & -.55 & .12 & .30 \\
\hline 21. Eu trabalharia com tranquilidade em um necrotério. & -.05 & -.07 & .04 & -.60 & .22 & .36 \\
\hline \multicolumn{7}{|l|}{ Fator 5: "Eventos mórbidos em noticiários/acidentes" } \\
\hline 06. Imagens de vítimas ensanguentadas deveriam... & .13 & -.17 & -.14 & -.15 & .45 & .20 \\
\hline 09. Gosto de acompanhar notícias de crimes... & .20 & .08 & .23 & -.12 & .45 & .20 \\
\hline 04. Tenho costume de assistir a programas ou ler... & -.04 & .10 & .10 & -.04 & .80 & .64 \\
\hline 11. Quando estou na estrada e vejo um acidente... & .16 & .07 & -.21 & -.20 & .57 & .32 \\
\hline Valor Próprio & 7.40 & 2.11 & 2.03 & 1.86 & 1.64 & \\
\hline Variância (\%) & 24.66 & 7.05 & 6.76 & 6.21 & 5.48 & \\
\hline Alfa de Cronbach dos Fatores & .79 & .73 & .73 & .82 & .62 & \\
\hline Alfa de Cronbach da Escala Total & .69 & & & & & \\
\hline
\end{tabular}

Nota. O conteúdo dos itens e a versão completa da escala podem ser solicitados à primeira autora

Actualidades en Psicología, 35(131), 2021, 103-118 
A solução final, composta por trinta itens e cinco fatores explicou 50.16\% da variância total e obteve alfa de Cronbach de .69. O primeiro fator reuniu dez itens de "Eventos Mórbidos Reais" com alfa de .79; o segundo fator reuniu sete itens de "Aversão a Eventos Mórbidos" e alfa de .73; o terceiro fator agrupou quatro itens de "Eventos mórbidos fictícios" com alfa de .73; o quarto fator reuniu cinco itens de "Eventos Mórbidos Médicos" com alfa de .82; e, por fim, o quinto fator agrupou os quatro itens de "Eventos Mórbidos em noticiários/acidentes" com alfa de .62.

\section{Discussão parcial}

Zuckerman e Litle (1986), ao criarem a escala de Curiosidade por Eventos Mórbidos (CAME), almejavam desenvolver uma medida que os permitissem compreender melhor o traço de personalidade descrito, assim como sua associação com os outros, como a busca de sensação. Desde sua publicação, outros estudos fizeram uso da medida proposta (Pinkerton \& Zhou, 2008; McDaniel et al., 2007; Weaver III et al., 1993), chegando a ser adaptada e traduzida para aplicação na Espanha (Aluja-Fabregat, 2000).

As estatísticas empregadas em prol de analisar o construto por Zuckerman e Litle (1986) não objetivaram analisar os parâmetros psicométricos da medida em si, de forma que ela foi analisada em função da atitude expressa nos seus itens, ou seja, os itens cujo conteúdo descrevia uma atitude positiva em relação ao evento mórbido relatado eram agrupados, formando a pontuação total da pessoa na CAME, ao passo que os itens que expressavam atitudes negativas se agrupavam formando o escore total em aversão a eventos mórbidos.

Por outro lado, esse artigo pretende contribuir com duas lacunas teóricas, o que justifica o uso da metodologia empregada. A primeira lacuna se configura em contribuir com a compreensão do fenômeno em contexto brasileiro e a segunda em compreender a estrutura fatorial atrelada ao construto estudado.

No contexto brasileiro, identifica-se o problema de ter acesso aos eventos mórbidos somente dentro de temas específicos (Frota, 2013; Santos et al., 2015). No entanto, efetivamente, não foi possível acessar nenhuma publicação que utilizasse os termos "Eventos Mórbidos" ou "Curiosidade por Eventos Mórbidos", ou ainda que se referisse ao fenômeno como um traço de personalidade.

Quanto à segunda questão, tem-se em vista que, na literatura atual, os estudos acerca do tema são escassos e fragmentados. A fragmentação se refere à ampla gama de metodologias empregadas e às temáticas específicas. Seja essa metodologia experimental (Lynch \& Martins, 2015); estudos de neuroimagem (Oosterwijk et al., 2015); avaliando a preferência por imagens neutras, positivas ou negativas visando entender essa preferência pelo estímulo negativo (Oosterwijk, 2017); ou, ainda, empregando a análise fenomenológica interpretativa (Fox, 2013). Dentro desse escopo, o construto vem sendo compreendido como unidimensional pelas pesquisas citadas. Porém, a própria perspectiva de estudar o tema em função de diversos tópicos específicos pode sugerir a existência de fatores dos "Eventos Mórbidos".

Tendo em vista os objetivos propostos, como resultado da análise de componentes principais, obteve-se uma estrutura fatorial de cinco fatores, cujos parâmetros psicométricos foram índices de satisfatórios a excelentes (Pasquali, 2010). Especificamente, todos os itens da medida saturaram com carga fatorial acima do ponto de corte de $|0.30|$ indicado pela literatura (Hair et al., 2009) em um dos fatores da medida. No entanto, aponta-se 
que alguns itens saturaram em mais de um fator. Tal fato pode indicar a proximidade entre os fatores, corroborando, inclusive, com a hipótese unifatorial assumida pela maioria das pesquisas. A variância explicada pelo instrumento foi de $50.16 \%$, estando dentro do esperado. Os parâmetros de confiabilidade foram, em sua maioria, acima do indicado para pesquisas (Pasquali, 2010), o qual é um Alpha de Cronbach acima de |0.70|. A exceção ficou a cargo do fator cinco "Eventos mórbidos em noticiários/acidentes", que apresentou alfa de |0.62|. Ressalta-se que o parâmetro não ficou tão abaixo do ideal, sendo comum que resultados como esse não interfiram com o prosseguimento de pesquisas e com o uso de instrumentos (Hair et al., 2009).

Com base no exposto, levantou-se a hipótese de que o construto Atração por Eventos Mórbidos poderia apresentar uma estrutura fatorial como a encontrada, de cinco fatores. Julgou-se pertinente então a realização de um segundo estudo com vistas a averiguar a estrutura fatorial hipotetizada da escala por meio de uma análise fatorial confirmatória e, ainda, analisar a relação da Atração por Eventos Mórbidos, em contexto brasileiro, com seus correlatos (Big Five e busca de sensações).

\section{Estudo 2}

\section{Análise confirmatória da EMAEM e relação com seus correlatos}

\section{Método}

\section{Amostra}

Participaram do estudo 609 pessoas da população geral do país, cuja maioria era do sexo feminino (60.3\%), heterossexual (77.3\%), solteira (59.9\%), católica (46.3\%), com idades variando entre 18 e 87 anos $(M=32.47 ; D P=13.82)$ e com ensino superior incompleto (41.4\%).

\section{Instrumentos}

Como instrumentos para a análise, além da escala EMAEM e das questões sociodemográficas adotadas no estudo anterior, utilizou-se os seguintes instrumentos:

Inventário dos Cinco Grandes Fatores de Personalidade - Reduzido (IGFP-5). Proposto por John et al. (1991), traduzido e adaptado para o contexto brasileiro por Gouveia et al. (2009). Os cinco grandes fatores estudados são: "Abertura a Mudanças, Conscienciosidade, Extroversão, Amabilidade e Neuroticismo". Passos e Laros (2015) apresentaram a escala dos cinco grandes fatores de personalidade em versão reduzida utilizada nesse estudo, contando com 20 itens a serem respondidos em uma escala tipo Likert que varia de $1=$ discordo totalmente a 5 = concordo totalmente. Nesta amostra, a escala total obteve Alpha de Cronbach de .74 e os alfas dos fatores foram de .71; .67; .76; .63; e .75, respectivamente.

Escala Abreviada de Busca de Sensações (BSSS-8). Criada por Zuckerman et al. (1978), a fim de analisar o interesse em buscas por experiência e excitação ou aventura e emoção. A versão abreviada foi desenvolvida por Hoyle et al. (2002), contando com oito itens que devem ser respondidos em uma escala tipo Likert variando de $0=$ discordo totalmente a 4 = concordo fortemente. A confiabilidade (Alpha de Cronbach) variou entre .68 e .79 (Hoyle et al., 2002; Ferreira, 2009). Neste artigo, o coeficiente da escala foi de .75.

Procedimentos de coleta de dados

A coleta de dados ocorreu por meio da aplicação dos questionários impressos, a partir da colaboração de pesquisadores voluntários. Adotaram-se os mesmos procedimentos éticos do estudo 01. 
Procedimentos de análise de dados

Para as análises, inicialmente, utilizou-se o software SPSS 21. Foram calculadas estatísticas descritivas, comparações de médias (teste $t$ de Student para amostras independentes) e correlações $r$ de Pearson. Em seguida, por meio do software AMOS 21, buscou-se avaliar o ajuste dos dados aos modelos propostos. Nestas análises, considerou-se como entrada a matriz de covariância, tendo sido adotado o estimador ML (Máxima Verossimilhança) e, na comparação dos modelos, levou-se em conta os seguintes indicadores: a razão qui-quadrado por graus de liberdade $\left(\mathrm{X}^{2} / \mathrm{gl}\right)$, o Goodness-of-fit index (CFI), o Adjusted Goodness-of-fit-index (AGFI) e a Root mean square error of approximation (RMSEA). Vale ressaltar que os modelos com $\chi^{2} / g$ l entre 2 e 3 ou inferior, CFI igual ou superior a .90, AGFI igual ou superior a .90 e RMSEA de .08 ou menos podem ser considerados adequados (Byrne, 2001).

\section{Resultados}

Antes de apresentar os resultados, é importante ressaltar que, neste estudo, a análise dos modelos desconsiderou os itens que no primeiro estudo apresentaram cargas fatoriais acima de $|0.30|$ em mais de um fator. Em função disso, a escala final contou com 22 itens distribuídos como segue entre os fatores: "Eventos Mórbidos Reais" (itens 08, 14, 15, 16,17, 24, 27; $\alpha=.75$ ); "Aversão a Eventos Mórbidos" (itens 10, 20, 28, 30; $\alpha=.65$ ); "Eventos Mórbidos Fictícios" (itens 03, 22, 29; $\alpha=.69$ ); "Eventos Mórbidos Médicos" (itens 07, 21, 25, 26; $\alpha=.82$ ); e "Eventos Mórbidos em Noticiários/Acidentes" (itens 04, 06, 09, 11; $\alpha=.65$ ).

Com base nesta distribuição dos itens, efetuaram-se análises fatoriais confirmatórias, testando dois modelos específicos: um unifatorial, agrupando os 22 itens; e outro composto por cinco fatores, em que se considerou a estrutura proposta no Estudo 1. 0 modelo unifatorial da EMAEM apresentou índices de ajuste abaixo dos pontos de corte estabelecidos na literatura (razão $\chi^{2} / g$ l de 7,70, AGFI $=.74, \mathrm{CFI}=.62$ e RMSEA de .10 [IC90\% = .100 - .110]). No modelo de cinco fatores os índices de ajustes foram superiores, mas não atingiram níveis satisfatórios (razão $\chi^{2} / \mathrm{gl}$ de 3.18, $\mathrm{AGFI}=.89, \mathrm{CFI}=.88$ e RMSEA de $.06[$ [IC90\% $=.055-.065])$.

Em função desses resultados, levando em consideração a pertinência teórica, optouse por observar os índices de modificação e reespecificar o modelo a partir das conexões entre os erros e9 e e19; e7 e e17; e, por fim e13 e e21. Após a realização desse procedimento, os indicadores atestaram o ajuste do modelo de cinco fatores (razão $\mathrm{X}^{2} / \mathrm{gl}$ de 2.76, $\mathrm{AGFI}=.90, \mathrm{CFI}=.91 \mathrm{e}$ RMSE de .05 [IC90\% $\left.=.048-.059\right]$ ); todas as saturações foram diferentes de zero $(\lambda \neq 0 ; z>1.96, p<.05)$, variando entre .44 (Item 4. Tenho costume de assistir a programas ou ler notícias policiais) e .89 (Item 26. Gostaria de assistir a uma cirurgia em que o abdômen do paciente estivesse completamente aberto). A variância média extraída (VME) foi igual a .38 e a confiabilidade composta igual a .93, corroborando a consistência interna da EMAEM. A Figura 2 apresenta e estrutura do modelo proposto.

Uma vez que o julgamento a respeito da validade de um instrumento pode ser mais substancial, dependendo das estratégias de validação que são utilizadas (Cohen et al., 2014), além das análises empregadas para atestar a estrutura fatorial do instrumento, também se buscou avaliar evidências de validade convergente para EMAEM. Para tanto, considerou-se a relação existente entre os eventos mórbidos com a busca de sensações e com os cinco grandes fatores de personalidade (Big Five).

Actualidades en Psicología, 35(131), 2021, 103-118 


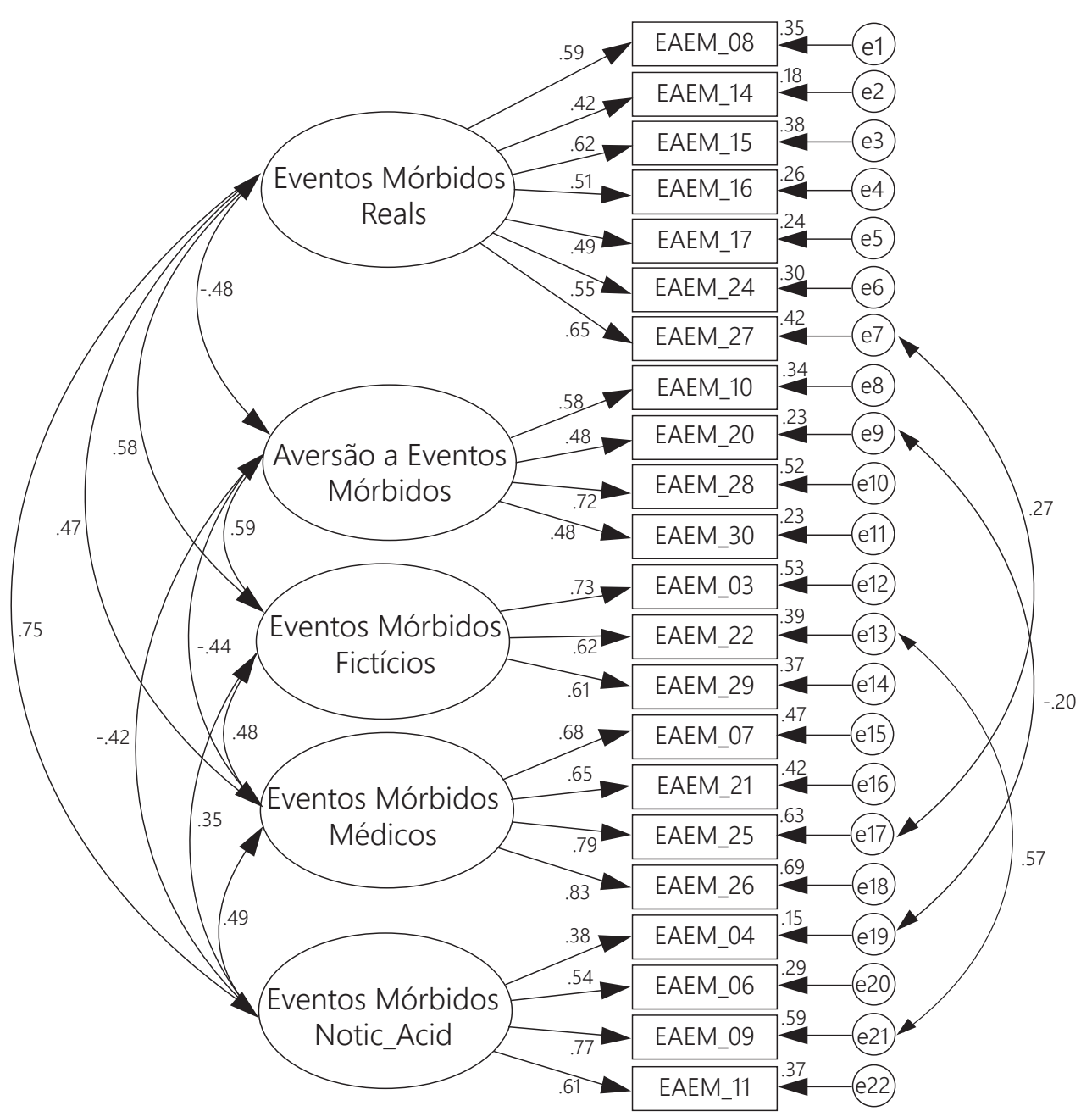

Figura 2. Análise Fatorial do modelo de cinco fatores para a EMAEM $(n=609)$

Em relação à busca de sensações, foram encontradas correlações significativas com todos os fatores da EMAEM, de forma que quanto maior a pontuação em busca de sensações, maior também foi a sua pontuação na EMAEM. O fator "Aversão a Eventos Mórbidos foi o único que obteve correlação negativa com a busca de sensações $(r=$ -.14, $p$ < .001). Os "Eventos Mórbidos Médicos" ( $r=.30, p<.001)$ e os "Eventos Fictícios" $(r=.33, p<.001)$ apresentaram as correlações mais fortes. Tais resultados podem ser observados na Tabela 2. 
Tabela 2

Correlações entre os fatores da EMAEM e os construtos correlatos $(n=609)$

\begin{tabular}{lccccccc}
\hline Variáveis & $M$ & $D P$ & \multicolumn{5}{c}{ Fatores EMAEM } \\
\cline { 3 - 8 } & & & $\mathrm{F} 1$ & $\mathrm{~F} 2$ & $\mathrm{~F} 3$ & $\mathrm{~F} 4$ & $\mathrm{~F} 5$ \\
\hline Busca de sen- & 2.68 & .79 & .24 & -.14 & .33 & .30 & .15 \\
sações & & & $(p<.001)$ & $(p<.001)$ & $(p<.001)$ & $(p<.001)$ & $(p<.001)$ \\
Abertura a & 3.70 & .71 & .02 & -.01 & .04 & .06 & .03 \\
mudança & & & $(.335)$ & $(.450)$ & $(.160)$ & $(.052)$ & $(.249)$ \\
Conscienciosidade & 3.98 & .61 & -.10 & .06 & -.13 & -.02 & .15 \\
& & & $(.006)$ & $(.055)$ & $(.001)$ & $(.338)$ & $(.000)$ \\
Extroversão & 3.50 & .79 & -.06 & .11 & -.10 & -.04 & .08 \\
& & & $(.064)$ & $(.002)$ & $(.005)$ & $(.134)$ & $(.025)$ \\
Amabilidade & 4.03 & .60 & -.25 & .15 & -.19 & -.05 & -.04 \\
& & & $(p<.001)$ & $(p<.001)$ & $(p<.001)$ & $(.098)$ & $(.149)$ \\
Neuroticismo & 3.26 & .90 & .13 & -.02 & .14 & .14 & .14 \\
& & & $(p<.001)$ & $(.287)$ & $(p<.001)$ & $(p<.001)$ & $(p<.001)$ \\
\hline
\end{tabular}

Nota. $\mathrm{F} 1=$ Eventos Mórbidos Reais $(M=1.78, D P=.88) ; F 2=$ Aversão a Eventos Mórbidos $(M=4.70, D P$ $=1.43) ; F 3=$ Eventos Mórbidos Fictícios $(M=3.12, D P=1.55) ; F 4=$ Eventos Mórbidos Médicos $(M=2.55$, $D P=1.54) ; F 5=$ Eventos Mórbidos em Noticiários/Acidentes $(M=2.36, D P=1.17)$.

No que tange à personalidade, especificamente ao modelo dos Big Five, o traço "Abertura a Mudanças" não apresentou correlações significativas com os fatores da EMAEM. Por outro lado, as pontuações em "Extroversão" se correlacionaram, positiva e significativamente, com a "Aversão a Eventos Mórbidos" ( $r=.11, p=.002$ ) e com "Eventos Mórbidos em Noticiários/Acidentes" ( $r=.08, p=.025)$ e, negativa e significativamente, com "Eventos Mórbidos Fictícios" ( $r=-.10, p=.005)$, enquanto que para os demais fatores não foram observadas correlações significativas. A "Conscienciosidade" se correlacionou de forma positiva com os "Eventos Mórbidos em Noticiários/Acidentes" ( $r=.15, p<$ .001) e negativa com "Eventos Mórbidos Fictícios" ( $r=-.13, p=.001)$. A "Amabilidade" apresentou correlação negativa com "Eventos Mórbidos Reais" $(r=-.25, p<.001)$ e "Eventos Mórbidos Fictícios" ( $r=-.19, p<.001)$ e, ainda, correlação positiva com a aversão a eventos mórbidos $(r=.15, p<.001)$. O traço neuroticismo, por sua vez, correlacionou-se de forma positiva com quatro dos fatores da EMAEM (correlações de $r=.14, p<.001 \mathrm{em}$ 3 desses fatores e $r=.13, p<.001$ em "Eventos Mórbidos Reais"), não sendo observada correlação significativa entre esse traço e a "Aversão a Eventos Mórbidos".

\section{Discussão geral}

O modelo unifatorial não demonstrou adequabilidade, endossando a proposta de um instrumento multifatorial, contemplando diferentes temáticas em cada fator (eventos mórbidos médicos, fictícios, entre outros). Desse modo, o presente estudo demonstra a possibilidade de utilizar um único instrumento geral para o estudo de diversos tipos de eventos mórbidos. 
A EMAEM demonstrou boa adequabilidade do modelo de cinco fatores, em especial após a inserção dos índices de modificação. Os índices de adequação do modelo estavam todos dentro dos parâmetros esperados pela literatura (Hair et al., 2009). Quanto aos índices de modificação utilizados, ressalta-se que os termos de erro apresentavam semelhanças no seu conteúdo, como segue:

A primeira díade se evidencia pelos seguintes itens: "Fico empolgado(a) quando assisto a espetáculos nos quais o artista corre o risco de morrer" e "Seria interessante assistir a uma autópsia (médicos examinando um cadáver aberto)". A análise semântica dos itens permite perceber que o conteúdo de ambos é voltado a ser espectador de um fenômeno mórbido. Não obstante, os itens "Sinto-me incomodado com programas que exibem violência" e "Tenho costume de assistir a programas ou ler notícias policiais", também apresentam semelhança nos conteúdos, no caso, relacionados a mídias que envolvem eventos mórbidos, seja por aproximação ou aversão. Por fim, os itens "Histórias de crimes e de assassinatos despertam a minha curiosidade" e "Gosto de acompanhar notícias de crimes que envolvem mortes" trazem semelhanças plausíveis pelo fato de explorarem o ato de conhecer os eventos mórbidos através de narrativas.

Para além da investigação da estrutura fatorial dos eventos mórbidos, também se averiguou sua relação com alguns construtos relevantes. Inicialmente, investigou-se a relação existente entre os eventos mórbidos e a busca de sensações. Como destacado, as correlações significativas e positivas foram percebidas em todos os fatores, salvo a "Aversão a Eventos Mórbidos", onde se encontrou uma correlação significativa e negativa. Ambos os resultados corroboram com estudos prévios (Zuckerman \& Litle, 1986; Lynch \& Martins, 2015).

Lynch e Martins (2015) apontam que as pessoas com alta busca de sensações possuem propensão ao envolvimento com atividades perigosas e comportamentos ilícitos; podese perceber, ainda, que a aversão ao tédio, que é uma das formas de operacionalização da busca de sensações (Hoyle et al., 2002), tem ligação direta com a definição dos eventos mórbidos. Além disso, é notório que os resultados são similares também ao estudo clássico de Zuckerman e Litle (1986). Oosterwijk (2017) alega ainda que essa relação está ligada à evocação de determinados estímulos evolutivos. Em relação à correlação negativa com "Aversão a Eventos Mórbidos", atribui-se esse resultado a características de segurança, inibição, conformidade e estabilidade (Ferreira, 2009), os quais trazem a concepção de repudiar sensações aversivas e encorajadoras de novas experiências, como os eventos mórbidos.

Em relação à personalidade, os resultados confirmam que a personalidade está ligada de forma significativa ao fenômeno da atração por eventos mórbidos (John, et al., 1991). No que concerne ao traço "Abertura a Mudanças", ele foi o único fator que não se correlacionou com nenhum fator da EMAEM. Estudos como os de Pimentel et al. (2014) demonstram que a "Abertura a Mudanças" encontra-se relacionada a aspectos dos eventos mórbidos, como gostar de filmes de terror. No entanto, a definição desse traço é destacada a partir de um interesse voltado para experiências culturais e intelectuais (Andrade, 2008), não incluindo apenas a ideia da busca de sensações, mas também uma série de outras características como valorização da estética e criatividade, sem especificar a preferência por nenhum gênero ou tipo de experiências culturais. Conjectura-se que a falta de relação esteja pautada nas demais características do traço de fato não serem correlatas aos eventos mórbidos e à não especificidade dos itens em relação aos tipos de experiências buscadas. Desse modo, na amostra pesquisada é possível que as pessoas que responderam se interessem por experiências novas, mas não relacionadas à temática dos eventos mórbidos. 
Já o traço de "Extroversão", caracterizado por fortes interações interpessoais, capacidade de alegrar-se e externalizar sentimentos, correlacionou-se positivamente com a "Aversão a Eventos Mórbidos" pelo fato de se aproximar de fenômenos mais reforçadores e que demonstram bem-estar. Ademais, tal traço se correlacionou negativamente com "Eventos Mórbidos Fictícios" e "Eventos Mórbidos em Noticiários/Acidentes", reforçando a ideia de que sujeitos com traços de extroversão buscam sensações voltadas à vitalidade e assertividade (Nunes \& Hutz, 2007).

Como esperado, o traço "Concienciosidade" não obteve correlações com os fatores "Eventos Mórbidos Reais", "Atração por Eventos Mórbidos" e "Eventos Mórbidos Médicos". Esse traço é caracterizado pela organização, persistência e disciplina (Nunes \& Hutz, 2007), e mostrou correlação significativa e positiva com "Eventos Mórbidos em Noticiários/Acidentes", a qual era esperada, visto que estar informado sobre os perigos e acidentes auxiliam os sujeitos com o autocuidado e a reduzir preocupações sobre seu ambiente. De fato, este achado corrobora o que Oosterwijk (2017) traz acerca de a atração por eventos mórbidos poder estar atrelada à busca de conhecimentos sobre o ambiente social. Além disso, "Conscienciosidade" correlacionou-se significativamente com o fator "Eventos Mórbidos Fictícios", porém com uma associação negativa, reforçando a concepção de que a conscienciosidade caracteriza-se, também, por uma evitação de acontecimentos e notícias fantasiosas que pouco fornecem informações importantes para comportamentos disciplinados (Andrade, 2008).

Com "Amabilidade", foi encontrada uma correlação significativa e positiva com o fator "Aversão a Eventos Mórbidos" e, negativa para os fatores "Eventos Mórbidos Reais" e "Eventos Mórbidos Fictícios". O resultado foi distinto do encontrado por Pimentel et al. (2014), que investigaram a preferência de estilos de filme de acordo com os cinco fatores da personalidade e seus resultados apontaram que aqueles que pontuavam mais em amabilidade preferiam filmes de terror (eventos mórbidos fictícios) em comparação a filmes de suspense.

No entanto, devido o traço ser normalmente definido por atitudes pró-sociais e empáticas (Silva et al., 2007), entende-se que tais pessoas tendem a afastar-se da ideia de presenciar fenômenos que ferem suas concepções de generosidade e interações interpessoais positivas, como normalmente é o caso dos eventos mórbidos (Andrade, 2008). De modo geral, compreende-se que sujeitos com estes traços se distanciam desses fenômenos, dos reais e fictícios principalmente, visto que os outros eventos, como médicos ou de noticiários/acidentes, podem estar associados ao ganho de informações (notícias) e ao entretenimento (seriados e videogames) e, ainda, apresentarem características mais relacionadas a estímulos positivos.

Pessoas com traços de neuroticismo em evidência podem se caracterizar por uma instabilidade emocional tendenciosa a transtornos psicológicos (Nunes \& Hutz, 2007). Todas as correlações observadas foram significativas e positivas, exceto pelo fator "Aversão a Eventos Mórbidos", para o qual não houve resultado significativo. Sujeitos com altas pontuações em "Neuroticismo" parecem também apresentar maior atração por eventos mórbidos por normalmente apresentar uma instabilidade que envolve irritação, frustração de realizações, melancolia e atração por afetos negativos, como tensão nervosa (Andrade, 2008).

De forma geral, os resultados encontrados neste estudo foram no mesmo sentido da literatura. Aponta-se como principal limitação a quantidade escassa de estudos 
sobre o tema. No entanto, compreende-se que há uma relevância teórica da temática devido a sua associação com um estilo de vida mais arriscado ou antissocial. Não obstante isso, os eventos mórbidos mostraram-se, de fato, relacionados com variáveis psicológicas importantes, como busca de sensações, comportamentos antissociais e traços de personalidade.

Outro avanço a ser pontuado é a elaboração de uma escala atualizada sobre a atração por eventos mórbidos que permite estudar o tema de forma geral e dentro de cinco temáticas específicas, facilitando a metodologia de estudos futuros e diminuindo a necessidade de expor o sujeito a condições experimentais aversivas. No mais, indica-se que estudos futuros façam uso de estatísticas mais robustas e de amostras representativas da população. Quanto aos correlatos dos eventos mórbidos, indica-se também que seja investigada sua associação com outros traços de personalidade, como o "Psicoticismo" e com outras variáveis psicológicas, como o "Bem-estar Subjetivo".

\section{Referências}

Agnieszka, P. (2018). Factors Affecting Viewer's Television Preferences: A Review. International Journal of Advances in Social Sciences and Humanities, 5(6), 1-6. http://ijassh.com/index.php/IJASSH/article/view/56

Aluja-Fabregat, A. (2000). Personality and curiosity about TV and films violence in adolescents. Personality and Individual Differences, 29(2), 379-392. https://doi. org/10.1016/S0191-8869(99)00200-7

Andrade, J. M. (2008). Evidências de Validade do Inventário dos Cinco Grandes Fatores de Personalidade para o Brasil [Tese de doutorado, Universidade de Brasília]. Repositório Institucional Da UNB. http://repositorio.unb.br/handle/10482/1751

Baumeister, R. F., Bratslavsky, E., Finkenauer, C., \& Vohs, K. D. (2001). Bad is stronger than good. Review of general psychology, 5(4), 323-370. https://doi.org/10.1037//1089-2680.5.4.323

Brasil. (2016). Pesquisa Brasileira de Mídia. Hábitos de consumo de mídia pela população brasileira. http://antigo.secom.gov.br/atuacao/pesquisa/lista-de-pesquisas-quantitativas-equalitativas-de-contratos-atuais/pesquisa-brasileira-de-midia-pbm-2016-1.pdf/

Browne, M. W., \& Cudeck, R. (1993). Alternative ways of assessing model fit. In K. A. Bollen \& J. S. Long (Eds.), Testing structural equation models (pp. 136-162). Sage.

Byrne, B. M. (2001). Structural Equation Modeling with AMOS, EQS, and LISREL: Comparative approaches to testing for the factorial validity of a measuring instrument. International Journal of Testing, 1(1), 55-86

Cohen, R. J., Swerdlik, M. E., \& Sturman, E. D. (2014). Testagem e Avaliação Psicológica: Introdução a Testes e Medidas. AMGH Editora.

Conselho Federal de Psicologia. (2000). Resolução CFP nº 016/2000 de 20 de dezembro de 2000. Dispõe sobre a realização de pesquisa em Psicologia com seres humanos. Código de Ética de Psicologia. http://site.cfp.org.br/wp-content/ uploads/2000/12/resolucao2000_16.pdf

Eysenck, H. J., \& Eysenck, M. W. (1985). Personality and individual differences: A natural Science Approach. Plenum Press.

Actualidades en Psicología, 35(131), 2021, 103-118 
Ferreira, J. F. K. S. (2009). Busca de sensações e dependência alcoólica: um estudo com doentes alcoólicos. [Dissertação]. https://sigarra.up.pt/fpceup/pt/pub_geral.pub_ view?pi_pub_base_id=29659

Fox, R. (2013). The psychology behind morbid reality: an interpretative phenomenological analysis of the fascination with blood, gore, injury, and death on the internet. Southampton Solent University, 1-26. https://e-space.mmu.ac.uk/576648/1/ Fox\%20(Rebecca)\%202013\%20(Southampton\%20Solent)\%20Qualitative.pdf

Frota, L. D. (2013). Pornografia, desigualdade de gênero e agressão sexual contra mulheres. Psicologia \& Sociedade, 25(3), 592-601. http://www.redalyc.org/articulo. oa?id=309329764013

Gouveia, V. V., Santos, W. S., Pimentel, C. E., Diniz, P. K. C., \& Fonseca, P. N. (2009). Questionário de comportamentos anti-sociais e delitivos: evidências psicométricas de uma versão reduzida. Psicologia: Reflexão e Crítica, 22(1), 20 28. https://dx.doi.org/10.1590/S0102-79722009000100004

Gruber, M. J., Gelman, B. D., \& Ranganath, C. (2014). States of curiosity modulate hippocampus-dependent learning via the dopaminergic circuit. Neuron, 84(2), 486-496. https://doi.org/10.1016/j.neuron.2014.08.060

Hair, J. F., Black, W. C., Babin, B. J., Anderson, R. E., \& Tatham, R. L. (2009). Análise multivariada de dados. Bookman.

Hayton, J. C., Allen, D. G., \& Scarpello, V. (2004). Factor retention decisions in exploratory factor analysis: A tutorial on parallel analysis. Organizational Research Methods, 7(2), 191-207. https://doi.org/10.1177/1094428104263675

Horvath, P., \& Zuckerman, M. (1993). Sensation seeking, risk appraisal, and risky behavior. Personality and Individual Differences, 14(1), 41-52. http://dx.doi. org/10.1016/0191-8869(93)90173-Z

Hoyle, R. H., Stephenson, M. T., Palmgreen, P., Lorch, E. P., \& Donohew, R. L. (2002). Reliability and validity of a brief measure of sensation seeking. Personality and Individual Differences, 32(3), 401-414. https://doi.org/10.1016/s0191-8869(01)00032-0

Hsee, C. K., \& Ruan, B. (2016). The Pandora effect: The power and peril of curiosity. Psychological science, 27(5), 659-666. https://doi.org/10.1177/0956797616631733

John, O. P., Donahue, E. M., \& Kentle, R. L. (1991). Big five inventory. PsycTESTS. https://doi. org/10.1037/t07550-000

Litman, J. (2005). Curiosity and the pleasures of learning: Wanting and liking new information. Cognition \& Emotion, 19(6), 793-814. https://doi.org/10.1080/02699930541000101

Lynch, T., \& Martins, N. (2015). Nothing to fear? An analysis of college students' fear experiences with video games. Journal of Broadcasting \& Electronic Media, 59(2), 298-317. https://doi.org/10.1080/08838151.2015.1029128

McDaniel, S. R., Lim, C., \& Mahan III, J. E. (2007). The role of gender and personality traits in response to ads using violent images to promote consumption of sports entertainment. Journal of Business Research, 60(6), 606-612. https://doi.org/10.1016/j.jbusres.2006.06.009

Narlla, H. (2015). Barra Pesada comemora 25 anos, consolidado como líder de audiência entre programas policiais. Tribuna do Ceará. http://web.archive.org/ 
web/20150724224510/http://tribunadoceara.uol.com.br/noticias/cotidiano-2/ barra-pesada-comemora-25-anos-consolidado-como-lider-de-audienciaentre-programas-policiais/

Nunes, C. S. S., \& Hutz, C. (2007). Construção e Validação da Escala Fatorial de Socialização no Modelo dos Cinco Grandes Fatores de Personalidade. Psicologia: Reflexão e Crítica, 20(1), 20-25. http://www.redalyc.org/html/188/18820104/

Oosterwijk, S. (2017). Choosing the negative: A behavioral demonstration of morbid curiosity. PloSone, 12(7), 1-20. https://doi.org/10.1371/journal.pone.0178399

Oosterwijk, S., Lindquist, K. A., Adebayo, M., \& Barrett, L. F. (2015). The neural representation of typical and atypical experiences of negative images: Comparing fear, disgust and morbid fascination. Social Cognitive and Affective Neuroscience, 11(1), 11-22. https://doi.org/10.1093/scan/nsv088

Pasquali, L. (2010). Instrumentação psicológica: Fundamentos e práticas. Artmed.

Passos, M. F. D., \& Laros, J. A. (2015). Construção de uma escala reduzida de Cinco Grandes Fatores de personalidade. Avaliação Psicológica, 14(1), 115-123. http://pepsic.bvsalud. org/scielo.php?script=sci_arttext\&pid=\$1677-04712015000100014\&lng=pt\&tIng=pt

Pimentel, C. E., Ferreira, D. C. S., Vargas, M. M., Maynart, V. A. P., \& Mendonça, D. C. (2014). Preferência por estilos de filmes e suas diferenças nos cinco fatores de personalidade. Pesquisas e práticas psicossociais, 9(2), 233-244. http://pepsic. bvsalud.org/pdf/ppp/v9n2/09.pdf

Pinkerton, K., \& Zhou, S. (2008). Effects of morbid curiosity on perception, attention, and reaction to bad news. The University of Alabama McNair Journal, 129143. https://pdfs.semanticscholar.org/89af/0088a76c0a373e38a0911383e239 abca3995.pdf

Roberti, J. W. (2004). A review of behavioral and biological correlates of sensation seeking. Journal of research in personality, 38(3), 256-279. https://doi.org/10.1016/S00926566(03)00067-9

Santos, E. S., Santos, R. F., \& Ribeiro, M. (2015). Jogando com as máquinas: relação entre videogames violentos e cognições agressivas. Psicologia e Saber Social, 4(2), 246264. https://doi.org/10.12957/psi.saber.soc.2015.16416

Silva, R. S., Schlottfeldt, C. G., Rozenberg, M. P., Santos, M. T., \& Lelé, A. J. (2007). Replicabilidade do modelo dos cinco grandes fatores em medidas de personalidade. Mosaico: estudos em psicologia, 1(1), 37-49. http://www.academia.edu/3264398/Replicabilidade_do_ modelo_dos_cinco_grandes_fatores_em_medidas_de_personalidade

Weaver III, J. B., Brosius, H. B., \& Mundorf, N. (1993). Personality and movie preferences: A comparison of American and German audiences. Personality and Individual Differences, 14(2), 307-315. https://doi.org/10.1016/0191-8869(93)90128-P

Zuckerman, M. (2007). The sensation seeking scale V (SSS-V): Still reliable and valid. Personality and Individual Differences, 43(5), 1303-1305. https://doi.org/10.1016/j.paid.2007.03.021

Zuckerman, M., \& Litle, P. (1986). Personality and curiosity about morbid and sexual events. Personality and Individual Differences, 7(1), 49-56. https://doi. org/10.1016/0191-8869(86)90107-8 
Zuckerman, M., Eysenck, S., \& Eysenck, H. J. (1978). Sensation Seeking Scale, Form V. PsycTESTS. https://doi.org/10.1037/t04065-000

Zuckerman, M., Kuhlman, D. M., Joireman, J., Teta, P., \& Kraft, M. (1993). A comparison of three structural models for personality: The Big Three, the Big Five, and the Alternative Five. Journal of personality and social psychology, 65(4), 757-768. https://doi. org/10.1037/0022-3514.65.4.757 